\title{
Dabrafenib Plus Trametinib for BRAF V600E-Mutant Non-small Cell Lung Cancer: A Patient Case Report
}

\author{
Janet $\operatorname{Pan}^{1}$ \\ Published online: 27 June 2019 \\ (c) The Author(s) 2019
}

\begin{abstract}
Dabrafenib plus trametinib is US Food and Drug Administration approved combination therapy for use in patients with $B R A F$ V600E-mutant non-small cell lung cancer, but information on use outside of clinical trials is limited. We report the case of a 70-year-old Asian woman (never smoker) who was diagnosed with lung adenocarcinoma in May 2014. Testing at diagnosis was negative for programmed death ligand 1 or EGFR, ALK, and ROS1 alterations. She was started on carboplatinpemetrexed-bevacizumab and maintenance bevacizumab but progressed in September 2015. Subsequently, she progressed on second-line nivolumab and third-line docetaxel. In March 2016, pleural fluid obtained at diagnosis tested positive for the $B R A F$ V600E mutation and she received dabrafenib plus trametinib. She experienced rapid tumor shrinkage and symptom improvement and became able to participate in regular daily activities with no notable adverse events. In December 2016, she died from a hemorrhagic stroke considered unrelated to treatment. In this heavily pretreated patient with non-small cell lung cancer, dabrafenib plus trametinib elicited an excellent response.
\end{abstract}

\section{Key Points}

This patient case demonstrates the potential for dabrafenib plus trametinib to provide robust antitumor activity in patients with heavily pretreated non-small cell lung cancer, including treatment with immune checkpoint inhibitors.

The report highlights the importance of broad molecular testing at diagnosis in non-small cell lung cancer to identify actionable oncogenic driver alterations and inform treatment decisions.

Janet Pan

drjanpan@gmail.com

1 Compassionate Cancer Care Inc, 11180 Warner Ave, Suite 351, Fountain Valley, CA 92708, USA

\section{Introduction}

Treatment of non-small cell lung cancer (NSCLC) has progressed rapidly in recent years from a histology-driven chemotherapy-led approach to personalized therapy for the subset of patients with targetable oncogenic alterations [1]. For example, patients receiving targeted agents for activating mutations in epidermal growth factor receptor (EGFR) and rearrangements in ALK receptor tyrosine kinase $(A L K)$ and ROS proto-oncogene 1 , receptor tyrosine kinase (ROS1) have been shown to have longer survival than patients without a driver mutation who receive chemotherapy [2]. These findings reflect current treatment guidelines, in which targeted therapies are listed as the first-line standard of care for patients with tumors harboring these mutations [3, 4].

BRAF is a serine/threonine kinase downstream of RAS in the RAS-RAF-MEK-ERK signaling pathway. Mutations in $B R A F$ lead to constitutive activation of this pathway, resulting in increased cellular growth and proliferation [5]. Activating mutations in $B R A F$ occur in approximately $2-4 \%$ of patients with NSCLC and are generally mutually exclusive from other driver mutations [2, 6, 7]. However, the clinical characteristics that define patients likely to harbor $B R A F$ mutations are not clear [6, 8]. The most common $B R A F$ mutation is $\mathrm{V} 600 \mathrm{E}$, which results in a glutamate substitution for valine at codon $600[9,10]$. Some 
studies have reported that $B R A F$ V600-mutated NSCLC is associated with shorter survival compared with $B R A F$ wild-type disease $[10,11]$. The unmet needs of patients with $B R A F$-mutant NSCLC were further demonstrated in a large French national program in which more than half of patients received only best supportive care as secondline therapy [6].

The BRAF V600 mutation is more common in advanced melanoma than in NSCLC, reported in approximately half of patients [9]. Targeting BRAF V600-mutant tumors with selective BRAF and MEK inhibitors is the current standard of care in patients with metastatic melanoma [12]. Dabrafenib, a BRAF inhibitor, plus trametinib, a MEK1/2 inhibitor, was the first US Food and Drug Administration (FDA)-approved treatment combination based on data from the pivotal COMBI-d trial [13]. More recently, BRAF and MEK inhibitors have been used to treat BRAF V600-positive NSCLC. Vemurafenib, a BRAF inhibitor, demonstrated clinical activity as monotherapy in $B R A F$ V600-positive unresectable metastatic NSCLS in a phase II basket study [14]. In 62 patients with $B R A F$ V600-mutant NSCLC, the overall response rate (ORR) was $37 \%$ (95\% confidence interval 25-50) with a disease control rate of $79 \%$ and a median progression-free survival of 6.5 months $(95 \%$ confidence interval 5.2-9.0).

Dabrafenib was evaluated as monotherapy and in combination with trametinib in patients with $B R A F$ V600Epositive metastatic NSCLC in a non-randomized, threecohort, single-arm, open-label phase II trial (NCT01336634) [15-17]. In this trial, patients who had previously progressed on one or more platinum-based chemotherapies who received the combination therapy had an ORR of $67 \%$ compared with an ORR of $33 \%$ in patients who received dabrafenib monotherapy. Patients in the third cohort received combination dabrafenib and trametinib as first-line therapy and showed an ORR of $64 \%$. The results from this study demonstrated the efficacy of combination targeted therapy in BRAF V600-positive metastatic NSCLC in both the first- and second-line setting. Although the rarity of $B R A F$ V600E mutations in NSCLC has precluded randomized clinical trials of targeted agents in this setting, dabrafenib plus trametinib was approved in June 2017 by the FDA for patients with BRAF V600E-mutant NSCLC.

Although there are limited clinical trial data describing the efficacy of BRAF plus MEK inhibition in patients with BRAF V600E-mutant NSCLC, outcomes in a realworld setting are useful to inform clinical practice. Here, we describe a patient with BRAF V600E-mutated NSCLC adenocarcinoma who was treated in a community-based setting. Despite disease progression with non-targeted therapy, she experienced substantial symptomatic improvement and tumor regression following later-line therapy with dabrafenib and trametinib.

\section{Case Presentation}

In May 2014, an Asian woman aged 70 years without any history of smoking and with minimal exposure to second-hand smoke presented with acute-onset dyspnea. She had been in good health, exercised regularly, and had no significant comorbidities. Positron emission tomography/computed tomography revealed pleural effusion encompassing most of the left lung and thickening of the left pleura. Increased metabolic activity in the right paratracheal lymph node and precarinal lymph node was also observed. Following biopsy, she was diagnosed with left lung adenocarcinoma (stage IVA, malignant left pleural effusion). There was no evidence of endobronchial lesions, but narrowing of the left lower lobe bronchus was observed. Magnetic resonance imaging revealed no brain metastases. Testing revealed that the tumor was EGFR wild type and negative for programmed death ligand 1 (PD-L1) and rearrangements in $A L K$ and ROS1 (further molecular testing, including assessment of $B R A F$ mutations, was not performed at this time).

In June 2014, first-line therapy was started, comprising standard doses of carboplatin, pemetrexed, and bevacizumab for 4 months, and the patient had a good response. Positron emission tomography/computed tomography in September 2014 revealed significant decreases in the left pleural effusion and in soft-tissue nodular densities throughout the left hemithorax and decreased metabolic activity. Compared with initial scans, reductions were observed in the left lower lobe mass (from $5.6 \times 2.9$ to $5.2 \times 1.8 \mathrm{~cm}$ ) and left upper field nodularity (from $8.7 \times 4.1$ to $3.1 \times 2.6 \mathrm{~cm}$ ). Metabolic activity in the right paratracheal and prevertebral lymph nodes had also decreased. In November 2014, the patient received maintenance therapy with bevacizumab (standard dose) for 9 months.

A positron emission tomography/computed tomography scan performed in September 2015 revealed progressive disease throughout the left chest, left pleura, and left supraclavicular lymph node. Second-line therapy was delayed because of the need for a repeat biopsy (confirming PD-L1 negative status), and in December 2015, nivolumab $240 \mathrm{mg}$ intravenously every 2 weeks was started; the patient received four doses up to January 2016. However, during nivolumab therapy, the patient experienced debilitating disease progression; she was bedridden in the hospital, struggled to talk because of severe dyspnea, and required oxygen therapy $(10 \mathrm{~L} / \mathrm{min})$. A CT scan of the chest, abdomen, and pelvis conducted in January 2016 revealed right pleural effusion and new ascites, consistent with metastatic spread into the omentum and mesentery. There was also evidence of new widespread disease in the axial skeleton and increased pericardial thickening. The 
patient then received two cycles of docetaxel during February and March 2016. However, despite dose reduction and growth factor support during cycle 2, treatment was complicated by neutropenic fever, progressive dyspnea, and abdominal distension.

In March 2016, pleural fluid obtained at the original diagnosis (May 2014) was tested for the BRAF V600E mutation (SNaPshot Multiplex polymerase chain reaction [primer extension-based method]; Integrated Oncology), and the results were positive, indicating an exon $15(1799 \mathrm{~T}>\mathrm{A})$ mutation. Based on this finding, off-label treatment with dabrafenib $150 \mathrm{mg}$ twice daily plus trametinib $2 \mathrm{mg}$ once daily was initiated in March 2016. Up to this time, the patient was bedridden and struggled to sit up (Eastern Cooperative Oncology Group performance status 4). However, within 4 weeks of initiating dabrafenib plus trametinib, marked improvements in her general well-being were observed. The patient became able to participate in all usual activities of daily living, including performing household tasks and shopping, and in the absence of strenuous activity, had no limitations (Eastern Cooperative Oncology Group performance status 1). Computed tomography scans performed in May 2016 and August 2016 revealed a substantial tumor reduction (Fig. 1). Furthermore, dabrafenib plus trametinib therapy was well tolerated, with no notable adverse events. In August 2016, the patient was hospitalized because of Clostridium difficile colitis and then again 2 months later as a result of a recurrent infection. It was not determined whether these infections were related to therapy. Except for these hospitalizations, the patient continued to maintain a good quality of life until December 2016, when she died suddenly following a large hemorrhagic stroke. No brain metastases (brain magnetic resonance imaging without contrast on 16 July, 2016 showed no evidence of intracranial disease) or other potential cancer-related events were implicated in her death.

\section{Discussion and Conclusions}

This case study describes a patient with BRAF V600Emutated NSCLC who experienced an excellent response to dabrafenib plus trametinib following several prior lines of treatment with non-targeted therapy and substantially debilitating disease progression. We believe these observations are
Fig. 1 Chest computed tomography scans at diagnosis and after treatment. Carina (a), aortic arch (b), and nipple (c)

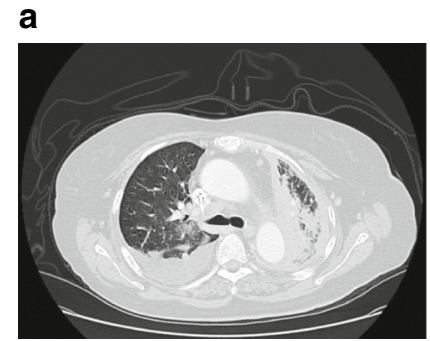

01-28-16

b

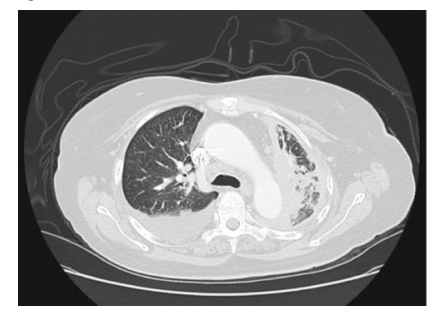

01-28-16

c

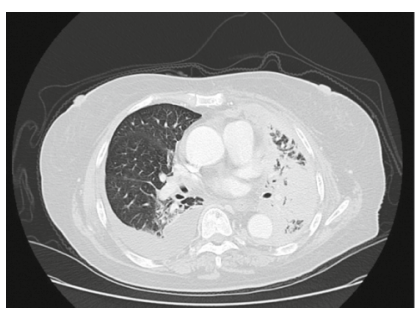

01-28-16

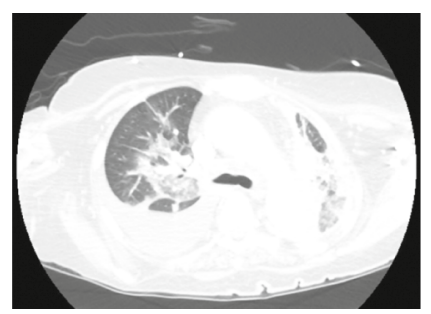

03-04-16

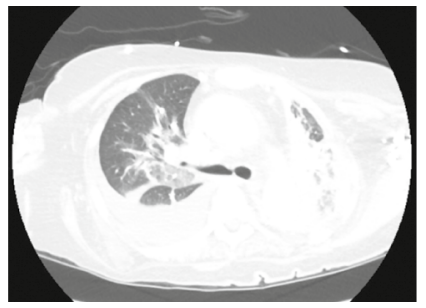

03-04-16

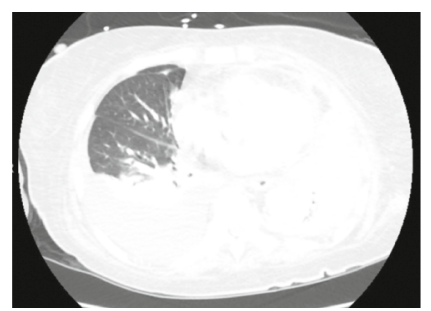

03-04-16

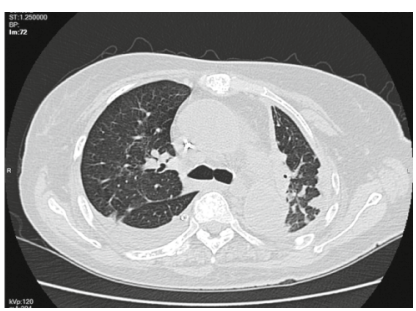

08-25-16

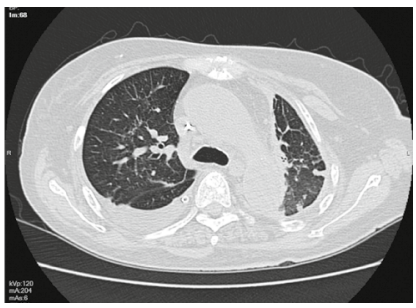

08-25-16

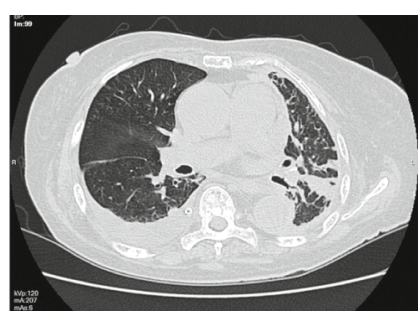

08-25-16 
noteworthy given the scarcity of data on treatment outcomes and unmet needs of patients with $B R A F$-mutant NSCLC.

This case study underscores the importance of testing for a broad range of actionable oncogenic driver mutations in patients with NSCLC. Although approximately two-thirds of NSCLC adenocarcinomas have a known oncogenic driver, several of these mutations, including $B R A F \mathrm{~V} 600 \mathrm{E}$, are relatively rare $(<5 \%)[2,6]$. Indeed, the National Comprehensive Cancer Network treatment guidelines for NSCLC endorse broader molecular profiling when diagnosing NSCLC to identify rare driver mutations for which effective drugs or clinical trials are available, thereby ensuring that patients receive the most appropriate treatments [12]. Use of dabrafenib plus trametinib in metastatic NSCLC is contingent on the detection of a BRAF V600E mutation using an FDA-approved test. Given the growing demands for molecular testing in limited biopsy material, expanded use of sequencing systems that can detect multiple driver mutations in a single test may help address the complex genetic landscape of NSCLC [4]. The FDA has recently approved two such tests that can be used to detect $B R A F$ V600E mutations and additional molecular drivers in patients with NSCLC, the Oncomine Dx Target Test (Thermo Fisher Scientific) and FoundationOne CDx (Foundation Medicine) [18].

In this case study, mutational testing for $B R A F \mathrm{~V} 600 \mathrm{E}$ preceded the FDA direction on companion diagnostics in NSCLC. Mutational testing for $B R A F$ was not included in the initial diagnostic assessment, and the mutation was instead detected almost 2 years later in a pleural effusion sample obtained at diagnosis. Assessment of $B R A F$ mutation status at diagnosis could have resulted in earlier treatment with dabrafenib plus trametinib, which could have potentially prolonged the duration of response and symptom control. Nevertheless, as fourth-line treatment, dabrafenib plus trametinib resulted in impressive reductions in tumor size and substantial improvements in quality of life. These treatment benefits were maintained for 9 months, until her sudden death as a result of a hemorrhagic stroke, which was considered unrelated to NSCLC or treatment. The rapid symptomatic improvement that this patient experienced was in line with reports from the clinical evaluation of the combination in patients with previously treated $B R A F$ V600Emutant metastatic NSCLC, which demonstrated a median time to radiographic response of 6 weeks, represented on this patient's first post-baseline scan [15].

To date, limited data are available to inform the optimal sequencing of agents in BRAF V600E-mutated NSCLC. In an open-label non-comparative study, dabrafenib plus trametinib demonstrated similar clinical benefit as both a first-line and later-line therapy $[15,16]$. These findings indicate that physicians have some flexibility regarding treatment strategies that may accommodate the needs of individual patients and local molecular testing protocols. In this case study, the immune checkpoint inhibitor nivolumab was administered as second-line therapy, but the patient experienced rapid disease progression. In the phase III CheckMate 057 trial, second-line nivolumab was associated with better efficacy outcomes compared with docetaxel, and this difference was greatest in patients with NSCLC whose tumors expressed PD-L1 [19]. Although the present case study provides evidence that response to dabrafenib plus trametinib is possible following treatment with immune checkpoint inhibitors, further research is required to determine the position of dabrafenib plus trametinib compared with platinum-based chemotherapy and immunotherapy options in treatment sequencing. Nevertheless, in view of the clinically significant and rapid response observed with dabrafenib plus trametinib in this case study, it may be reasonable to sequence BRAF plus MEK-targeted therapy ahead of immune checkpoint inhibition in patients with $B R A F$ V600E-mutated NSCLC, particularly in those with PD-L1-negative tumors.

Acknowledgements Editorial assistance was provided by Michael Demars, Ph.D., CMPP (ArticulateScience LLC), and was funded by Novartis Pharmaceuticals Corporation.

\section{Compliance with Ethical Standards}

Funding Editorial assistance in the drafting of this case report was funded by Novartis Pharmaceuticals Corporation.

Conflict of interest Janet Pan has no conflicts of interest that are directly relevant to the content of this article.

Authors' contributions JP treated the patient reported in this case study, drafted the manuscript, and approved the final version.

Ethics approval Not applicable.

Consent to participate Not applicable.

Consent for publication The patient's family provided written informed consent to publish this case report and any accompanying images.

Data availability The patient data reported in this study are not publicly available because of patient privacy but may be obtained from the corresponding author upon reasonable request.

Open Access This article is distributed under the terms of the Creative Commons Attribution-NonCommercial 4.0 International License (http://creativecommons.org/licenses/by-nc/4.0/), which permits any noncommercial use, distribution, and reproduction in any medium, provided you give appropriate credit to the original author(s) and the source, provide a link to the Creative Commons license, and indicate if changes were made. 


\section{References}

1. Mascaux C, Tomasini P, Greillier L, Barlesi F. Personalised medicine for nonsmall cell lung cancer. Eur Respir Rev. 2017. https:// doi.org/10.1183/16000617.0066-2017.

2. Kris MG, Johnson BE, Berry LD, Kwiatkowski DJ, Iafrate AJ, Wistuba II, et al. Using multiplexed assays of oncogenic drivers in lung cancers to select targeted drugs. JAMA. 2014;311(19):1998-2006.

3. Ettinger DS, Wood DE, Aisner DL, Akerley W, Bauman J, Chirieac LR, et al. Non-small cell lung cancer, version 5. 2017. NCCN clinical practice guidelines in oncology. J Natl Compr Cancer Netw. 2017;15(4):504-35.

4. Novello S, Barlesi F, Califano R, Cufer T, Ekman S, Levra MG, ESMO Guidelines Committee, et al. Metastatic non-small-cell lung cancer: ESMO clinical practice guidelines for diagnosis, treatment and follow-up. Ann Oncol. 2016;27(Suppl. 5):v1-27.

5. Vultur A, Villanueva J, Herlyn M. Targeting BRAF in advanced melanoma: a first step toward manageable disease. Clin Cancer Res. 2011;17(7):1658-63.

6. Barlesi F, Mazieres J, Merlio JP, Debieuvre D, Mosser J, Lena H, Biomarkers France Contributors, et al. Routine molecular profiling of patients with advanced non-small-cell lung cancer: results of a 1-year nationwide programme of the French Cooperative Thoracic Intergroup (IFCT). Lancet. 2016;387(10026):1415-26.

7. Tissot C, Couraud S, Tanguy R, Bringuier PP, Girard N, Souquet PJ. Clinical characteristics and outcome of patients with lung cancer harboring BRAF mutations. Lung Cancer. 2016;91:23-8.

8. Baik CS, Myall NJ, Wakelee HA. Targeting BRAF-mutant nonsmall cell lung cancer: from molecular profiling to rationally designed therapy. Oncologist. 2017;22(7):786-96.

9. Davies H, Bignell GR, Cox C, Stephens P, Edkins S, Clegg S, et al. Mutations of the BRAF gene in human cancer. Nature. 2002;417(6892):949-54.

10. Villaruz LC, Socinski MA, Abberbock S, Berry LD, Johnson $\mathrm{BE}$, Kwiatkowski DJ, et al. Clinicopathologic features and outcomes of patients with lung adenocarcinomas harboring BRAF mutations in the Lung Cancer Mutation Consortium. Cancer. 2015;121(3):448-56.

11. Marchetti A, Felicioni L, Malatesta S, Grazia Sciarrotta M, Guetti L, et al. Clinical features and outcome of patients with non-small-cell lung cancer harboring BRAF mutations. J Clin Oncol. 2011;29(26):3574-9.

12. National Comprehensive Cancer Center. NCCN clinical practice guidelines in oncology. Non-Small Cell Lung Cancer. V5. 2019. https://www.nccn.org/professionals/physician_gls/pdf/nscl.pdf. Accessed 26 Jun 2019.

13. Long GV, Stroyakovskiy D, Gogas H, Levchenko E, de Braud F, Larkin J, et al. Dabrafenib and trametinib versus dabrafenib and placebo for Val600 BRAF-mutant melanoma: a multicentre, double-blind, phase 3 randomised controlled trial. Lancet. 2015;386(9992):444-51.

14. Subbiah V, Gervais R, Riely GJ, Hollebecque A, Blay J, Felip E, et al. Efficacy of vemurafenib in patients (pts) with non-small cell lung cancer (NSCLC) with BRAFV600 mutation. J Clin Oncol. 2017;35(Suppl.):abstract 9074.

15. Planchard D, Besse B, Groen HJ, Souquet PJ, Quoix E, Baik CS, et al. Dabrafenib plus trametinib in patients with previously treated BRAF(V600E)-mutant metastatic non-small cell lung cancer: an open-label, multicentre phase 2 trial. Lancet Oncol. 2016;17(7):984-93.

16. Planchard D, Smit EF, Groen HJM, Mazieres J, Besse B, Helland A, et al. Dabrafenib plus trametinib in patients with previously untreated BRAF(V600E)-mutant metastatic non-smallcell lung cancer: an open-label, phase 2 trial. Lancet Oncol. 2017;18(10):1307-16.

17. Planchard D, Besse B, Kim TM, Quoix EA, Souquet PJ, Mazieres J, et al. Updated survival of patients (pts) with previously treated BRAF V600E-mutant advanced non-small cell lung cancer (NSCLC) who received dabrafenib (D) or D+ trametinib (T) in the phase II BRF113928 study. J Clin Oncol. 2017;35(Suppl.):abstract 9075.

18. US Department of Health and Human Services, US Food and Drug Administration. List of cleared or approved companion diagnostic devices (in vitro and imaging tools). https://www.fda.gov/ MedicalDevices/ProductsandMedicalProcedures/InVitroDiagnost ics/ucm301431.htm. Accessed 18 Dec 2017.

19. Borghaei H, Paz-Ares L, Horn L, Spigel DR, Steins M, Ready NE, et al. Nivolumab versus docetaxel in advanced nonsquamous non-small-cell lung cancer. N Engl J Med. 2015;373(17):1627-39. 